CUPhys/16/2006

\title{
Spin-spin Correlation in Some Excited States of Transverse Ising Model
}

\author{
Anjan Kumar Chandra and Subinay Dasgupta \\ Department of Physics, University of Calcutta, \\ 92 Acharya Prafulla Chandra Road, \\ Calcutta 700009, India.
}

(Dated: November 7, 2018)

\begin{abstract}
We consider the transverse Ising model in one dimension with nearest-neighbour interaction and calculate exactly the longitudinal spin-spin correlation for a class of excited states. These states are known to play an important role in the perturbative treatment of one-dimensional transverse Ising model with frustrated second-neighbour interaction. To calculate the correlation, we follow the earlier procedure of Wu, use Szego's theorem and also use Fisher-Hartwig conjecture. The result is that the correlation decays algebraically with distance $(n)$ as $1 / \sqrt{ } n$ and is oscillatory or non-oscillatory depending on the magnitude of the transverse field.
\end{abstract}

PACS numbers: 05.50.+q, 75.10.Pq, 02.30.Tb

\section{INTRODUCTION}

Transverse Axial Next-Nearest Neighbour Ising (TANNNI) model has attracted much attention recently as a simple quantum Ising model with frustration. In one dimension, it is defined by the Hamiltonian [1, 2, 3]

$$
\mathcal{H}_{A}=-J \sum_{j=1}^{N}\left(s_{j}^{z} s_{j+1}^{z}-\kappa s_{j}^{z} s_{j+2}^{z}\right)-\Gamma \sum_{j=1}^{N} s_{j}^{x}
$$

where $s_{j}^{z}$ and $s_{j}^{x}$ are the Pauli matrices $\sigma_{z}$ and $\sigma_{x}$ at the site $j, J$ is the strength of the nearest neighbour ferromagnetic interaction, $\kappa$ is the frustration parameter and $\Gamma$ is the transverse field. The phase diagram of the ground state of this model in the $\kappa$ - $\Gamma$ plane shows interesting features and is a matter of much controversy and investigation. Recently, we have shown [4] that the ground state of the Hamiltonian $\mathcal{H}_{A}$ is related to a class of excited states of a much simpler Hamiltonian obtained by removing the frustration (i.e. setting $\kappa=0$ ),

$$
\mathcal{H}=-J \sum_{j=1}^{N} s_{j}^{z} s_{j+1}^{z}-\Gamma \sum_{j=1}^{N} s_{j}^{x} .
$$

This Hamiltonian defines what is called transverse Ising model (in one dimension) and is the first quantum Ising model to be solved exactly $([\underline{5},[6]$, see also [1, 7] $)$.

All the eigenvalues and eigenfunctions of the Hamiltonian $\mathcal{H}$ for transverse Ising model can be derived exactly $\left[1,[5,6,6]\right.$. The $2^{N}$ number of energy eigenvalues are

$$
E=2 \Gamma \sum_{k} \xi_{k} \Lambda_{k}
$$

where $\Lambda_{k}=\sqrt{ }\left(\lambda^{2}+2 \lambda \cos k+1\right), \lambda=J / \Gamma, \xi_{k}$ may be 0 , \pm 1 and $k$ runs over $N / 2$ equispaced values in the interval 0 to $\pi$. The ground state is obviously

$$
E_{0}=-2 \Gamma \sum_{k} \Lambda_{k}
$$

corresponding to $\xi_{k}=-1$ for all $k$ and the excited states correspond to the other distributions of $\xi_{k}$ values. The spin-spin correlation function in the longitudinal direction

$$
C^{z}(n) \equiv<s_{j}^{z} s_{j+n}^{z}>-<s_{j}^{z}>^{2}
$$

has also been calculated exactly [5, 6] for the Hamiltonian $\mathcal{H}$ in the ground state; it decays exponentially with distance at $\lambda \neq 1$ and algebraically (with exponent $=$ $1 / 4$ ) at $\lambda=1$ (the critical point). As for the excited states, the correlation was first studied by McCoy [8]. The specific quantity calculated was the correlation at a temperature $T>0$ for the XY model (which in turn can be mapped onto the transverse Ising model). The correlation was found to decay exponentially with distance except when $T=0$ and $\lambda=1$.

The basic motivation of the paper is that (as mentioned above) the ground state of the Transverse Axial NextNearest Neighbour Ising Hamiltonian $\mathcal{H}_{A}$ in the region of interest (namely, $\kappa \sim 0.5, \Gamma \sim 0$, see ref. [4]) is related to a particular set of excited states of the transverse Ising Hamiltonian $\mathcal{H}$. This states have

$$
\xi_{k}=\left\{\begin{aligned}
-1 & \text { for } k=0 \text { to } \phi \text { (unexcited) } \\
1 & \text { for } k=\phi \text { to } \pi \text { (excited) }
\end{aligned}\right.
$$

It is thus important to study these states for transverse Ising model, especially, to calculate in these states, the longitudinal spin-spin correlation $C^{z}(n)$ for large $n$. In this paper we provide exact calculation for this quantity. Our result is that for these states, the correlation decays algebraically with exponent $1 / 2$ for all values of $\lambda \neq 1$, provided $\phi$ is neither 0 or $\pi$ (some levels are excited, but not all). This communication does not cover the case of $\lambda=1$.

Our results do not contradict the results obtained by McCoy, since at $T>0$, not only the excited states described by Eq. (5) but all possible excited states contribute to the correlation. Thus, any state with energy $E$ would provide a contribution by an amount proportional to $\exp \left(-E / k_{B} T\right)\left(k_{B}\right.$ is the Boltzmann constant). 
If the states with exponentially decaying correlation be dominant, the resultant correlation will not be algebraic.

Instead of calculating $C^{z}(n)$ directly, we shall rather calculate the quantity

$$
\sigma^{z}(n) \equiv<s_{j}^{z} s_{j+n}^{z}>
$$

We shall see that for the excited states discussed in this paper (see Eq. (5)),

$$
\lim _{n \rightarrow \infty} \sigma^{z}(n)=0
$$

so that $\left\langle s_{j}^{z}>\right.$ is also zero and $C^{z}(n)=\sigma^{z}(n)$. To evaluate the quantity $\sigma^{z}(n)$ for large $n$, we note that this quantity can be expressed as an $n \times n$ Toeplitz determinant $[\underline{5}, 6]$

$$
\operatorname{det}_{n}(\lambda) \equiv\left|\begin{array}{ccccc}
G_{0} & G_{-1} & G_{-2} & \cdots & G_{-n+1} \\
G_{1} & G_{0} & G_{-1} & \cdots & G_{-n+2} \\
G_{2} & G_{1} & G_{0} & \cdots & G_{-n+3} \\
\cdots \cdots & & & & \\
G_{n-1} & G_{n-2} & G_{n-3} & \cdots & G_{0}
\end{array}\right|
$$

where the elements are given by

$$
G_{m}=\frac{1}{\pi}\left[\int_{k=0}^{\phi}-\int_{k=\phi}^{\pi}\right] \frac{d k}{\Lambda_{k}}[\cos (k m-k)+\lambda \cos (k m)]
$$

In fact, Pfeuty [6] has given the expression for $G_{m}$ in the ground state and there this integral is from 0 to $\pi$. That expression has been modified here for the excited state following Lieb, Schultz and Mattis [9]. The standard prescription for studying the limiting $(n \rightarrow \infty)$ behaviour of a Toeplitz determinant is to apply Szego's Theorem in Fisher-Hartwig form [10, 11, 12, 13]. We shall also apply the same technique on the determinant given above.

In Secs. II and III we treat the cases $\lambda<1$ and $\lambda>1$ respectively and in Sec. IV we present some discussions.

\section{SPIN CORRELATIONS FOR $\lambda<1$}

We start this section with some analysis that is true both for $\lambda<1$ and $\lambda>1$. The first step is to observe that the expression (8) for the elements $G_{m}$ of the determinant can be written as,

$$
G_{m}=\frac{1}{2 \pi} \int_{0}^{2 \pi} d k \exp (-i m k) c(k)
$$

with the function $c(k)$ (called the generating function) given by,

$$
c(k)=\left\{\begin{aligned}
w & \text { for } 0<k<\phi \\
-w & \text { for } \phi<k<2 \pi-\phi \\
w & \text { for } 2 \pi-\phi<k<2 \pi
\end{aligned}\right.
$$

where

$$
w=\sqrt{\frac{\lambda+e^{i k}}{\lambda+e^{-i k}}}
$$

It should be mentioned that this generating function is similar to that for the classical two-dimensional Ising model [14] apart from the finite jumps at $k=\phi$ and $2 \pi-\phi$.

Till now, whenever we have come across a square root (Eqs. (3),(8),(11)) we have tacitly adopted the convention (like usual computer language compilers) that we shall always take the numerically positive root. However, since later (see Eqs.(16), (17) below) we shall need to integrate the logarithm of the generating function from 0 to $2 \pi$, let us choose, following the standard literature [15], the branch of square root for which this function is positive in the limit $k \rightarrow \pi$. Thus, we write the surd in Eq. (11) as

$$
w=\frac{\lambda+\cos k+i \sin k}{\sqrt{ }\left(1+\lambda^{2}+2 \lambda \cos k\right)}
$$

and observe that we should take the negative root for $\lambda>1$ and positive root for $\lambda<1$. Let us call the generating function evaluated with this new convention as $\bar{c}(k)$ which is related to the $c(k)$ of Eq.(10) by

$$
\bar{c}(k)=\left\{\begin{array}{r}
-c(k) \text { for } \lambda>1 \\
c(k) \text { for } \lambda<1
\end{array}\right.
$$

This $\bar{c}(k)$ when plugged in Eq. (9) leads to $\bar{G}_{m}$ and the Toeplitz determinant $\overline{\operatorname{det}}_{n}(\lambda)$ constructed from $\bar{G}_{m}$ can be evaluated by applying Szego's theorem on $\bar{c}(k)$. Obviously, this determinant is related to the $\operatorname{det}_{n}(\lambda)$ of Eq. (7) by

$$
\overline{\operatorname{det}}_{n}(\lambda)=\left\{\begin{aligned}
(-1)^{n} \operatorname{det}_{n}(\lambda) & \text { for } \lambda>1 \\
\operatorname{det}_{n}(\lambda) & \text { for } \lambda<1
\end{aligned}\right.
$$

The "index" of the generating function, defined as

$$
\text { Ind } \bar{c}(k)=\frac{1}{2 \pi i}[\log \bar{c}(2 \pi)-\log \bar{c}(0)]
$$

is 0 for $\lambda<1$ but +1 for $\lambda>1$. Since Szego's theorem can be directly applied only when the index is zero, our job is somewhat simpler for $\lambda<1$, compared to the same for $\lambda>1$.

We shall be confined to the case of $\lambda<1$ henceforth. The generating function for this case is,

$$
\bar{c}(k)=\left\{\begin{aligned}
w & \text { for } 0<k<\phi \\
-w & \text { for } \phi<k<2 \pi-\phi \\
w & \text { for } 2 \pi-\phi<k<2 \pi
\end{aligned}\right.
$$

Let us now investigate whether the conditions required (other than zero index) for Szego's theorem to be valid are satisfied here or not. For $\phi=\pi$ (ground state, all modes unexcited), one can make binomial expansion of the surd in $w$ (Eq. (11)) and conclude that for any large positive integral value of $m$ (with $\lambda<1$ ),

$$
\left|\bar{G}_{m}\right| \propto \frac{\lambda^{m}}{\sqrt{m}}
$$




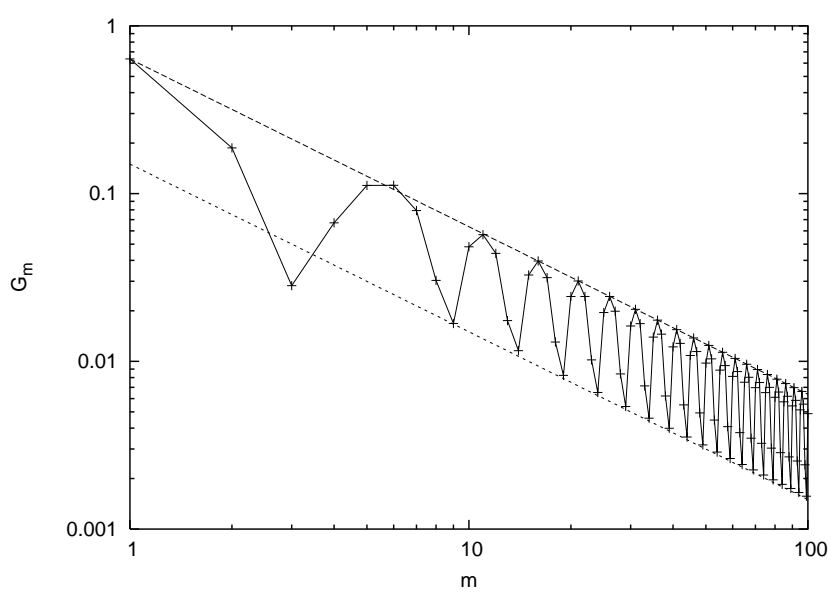

FIG. 1:

Plot of the elements of the Toeplitz determinant for $\phi=0.8$ and $\lambda=1.3$. The oscillations themselves depend on $\phi$ and $\lambda$ but the upper and the lower envelopes decay as $1 / m$ for all

$\lambda$ and $\phi$. The dotted lines are $0.635 / m$ and $0.150 / m$.

and

$$
\left|\bar{G}_{-m}\right| \propto \frac{\lambda^{m}}{m \sqrt{m}}
$$

Since $\bar{G}_{m}$ and $\bar{G}_{-m}$ both vanish exponentially with $m$, the conditions,

$$
\sum_{m=-\infty}^{\infty}\left|\bar{G}_{m}\right|<\infty \quad \text { and } \quad \sum_{m=-\infty}^{\infty}|m|\left|\bar{G}_{m}\right|^{2}<\infty
$$

are valid. Since $\bar{c}(k) \neq 0$ for all values of $k$, the Hirshmann conditions [16, 17] for the validity of Szego's theorem is satisfied. (However, it is not clear whether the Devinatz condition [16], namely, the continuity of

$$
\sum_{m=1}^{\infty}\left(\bar{G}_{m} e^{i m k}-\bar{G}_{-m} e^{-i m k}\right)
$$

is true.) For $\phi \neq \pi$, the asymptotic behaviour of $\left|\bar{G}_{m}\right|$ and $\left|\bar{G}_{-m}\right|$ depends on $\lambda$ and $\phi$ and is not easy to track analytically. Numerical study shows that both these quantities are oscillatory (Fig. 1). The upper and lower envelopes of the oscillatory curve decay as $1 / m$ for all $\lambda$ and $\phi$ while the wavelength and amplitude of oscillation depends on the specific values of $\lambda$ and $\phi$. It is thus difficult to analyse the validity of Hirshmann or Devinatz condition here. We do not proceed further with the question of validity of the conditions for applicability of Szego's theorem and rather choose to study the results obtained when we do apply the theorem anyway.

To apply Szego's theorem, the next step is to calculate the quantities $g_{0}$ and $g_{ \pm m}$ defined as

$$
g_{0}=\frac{1}{2 \pi} \int_{0}^{2 \pi} d k \ln \bar{c}(k)
$$

$$
g_{ \pm m}=\frac{1}{2 \pi} \int_{0}^{2 \pi} d k e^{\mp i m k} \ln \bar{c}(k),
$$

where $m$ is any positive integer. These quantities can be easily evaluated from Eq. (13), but we must be careful to choose the principal branch for $\ln \bar{c}(k)$, satisfying

$$
-i \pi<\ln \bar{c}(k)<i \pi .
$$

This criterion is satisfied if we express the logarithm as,

$$
\ln \bar{c}(k)=i k+\frac{1}{2} \ln \frac{1+\lambda e^{-i k}}{1+\lambda e^{i k}}+i \pi \delta
$$

with the phase factor

$$
\delta=\left\{\begin{aligned}
0 & \text { for } 0<k<\phi \\
-1 & \text { for } \phi<k<2 \pi-\phi \\
-2 & \text { for } 2 \pi-\phi<k<2 \pi .
\end{aligned}\right.
$$

By expanding the logarithm on the right hand side of Eq. (17a) in a series and performing a term-by-term integration, we now get

$$
g_{0}=0
$$

and

$$
g_{ \pm m}= \pm \frac{(-\lambda)^{m}}{2 m} \mp \frac{\cos (m \phi)}{m} .
$$

Next, we have to calculate the sum,

$$
\sigma=\sum_{m=1}^{\infty} m g_{m} g_{-m}
$$

Using the standard result [18]

$$
\sum_{n=1}^{\infty} \frac{1}{m} a^{m} \cos (m \alpha)=-\frac{1}{2} \ln \left(1-2 a \cos \alpha+a^{2}\right)
$$

(for $0<\alpha<2 \pi, a^{2} \leq 1$ ) one obtains,

$\sigma=\frac{1}{4} \ln \left(1-\lambda^{2}\right)+\frac{1}{2} \ln \left(\frac{2 \sin \phi}{1+2 \lambda \cos \phi+\lambda^{2}}\right)-\frac{1}{2} \sum_{m} \frac{1}{m}$.

The last sum is divergent. Following the conjecture of Fisher and Hartwig [11, 12, 13, 19], we replace the upper limit of the sum by $\zeta n$ to obtain

$$
\sum_{m=1}^{\zeta n} \frac{1}{m}=\gamma_{E}+\ln (\zeta n)
$$

where $\gamma_{E}$ is the Euler's constant $(=0.5772)$. The constant $\zeta$ is independent of $\lambda, \phi$ and $n$. Its numerical value could not be found analytically.

Szego's theorem now reads $10,11,12$

$$
\overline{\operatorname{det}}_{n}(\lambda)=\exp \left(n g_{0}+\sigma\right),
$$


which, on substitution of the expressions of $g_{0}$ and $\sigma$ leads to the final result

$$
C^{z}(n)=\overline{\operatorname{det}}_{n}(\lambda)=\frac{A \sqrt{\sin \phi}\left(1-\lambda^{2}\right)^{1 / 4}}{\sqrt{1+2 \lambda \cos \phi+\lambda^{2}}} \frac{1}{\sqrt{n}},
$$

where $A=(1 / \sqrt{\zeta / 2}) \exp \left(-\gamma_{E} / 2\right)$. We have numerically evaluated this constant to be 0.590 (which corresponds to $\zeta=3.226$ ) by computing determinants upto a size of $200 \times 200$. The correlation function $C^{z}(n)$ decays algebraically with distance without any oscillation.

\section{SPIN CORRELATIONS FOR $\lambda>1$}

We shall now evaluate the Toeplitz determinant of Eq. (7) for $\lambda>1$. Since the index of the generating function is not zero in this case (as mentioned above), Szego's theorem cannot be applied directly. One has to follow instead the method of $\mathrm{Wu}$ 20]. According to Eq. (8), the elements $G_{m}$ for some given value of $\lambda$ is related to the same for $1 / \lambda$ by,

$$
G_{m}(\lambda)=G_{-m+1}(1 / \lambda)
$$

Hence, the determinant for $\lambda=\lambda_{0}>1$, can be written as,

$$
\operatorname{det}_{n}\left(\lambda_{0}\right)=\left|\begin{array}{ccccc}
G_{1} & G_{0} & G_{-1} & \cdots & G_{-n+2} \\
G_{2} & G_{1} & G_{0} & \cdots & G_{-n+3} \\
\cdots \cdots & & & & \\
G_{n} & G_{n-1} & G_{n-2} & \cdots & G_{1}
\end{array}\right|_{\lambda=1 / \lambda_{0}}
$$

Let us consider the Wiener-Hopf system of equations,

$$
\sum_{m=0}^{n} G_{k-m}\left(1 / \lambda_{0}\right) x_{m}=\delta_{k, 0}
$$

for $k=0,1, \cdots n$. By Cramer's rule,

$$
x_{n}\left(1 / \lambda_{0}\right)=(-1)^{n} \frac{\operatorname{det}_{n}\left(\lambda_{0}\right)}{\operatorname{det}_{n+1}\left(1 / \lambda_{0}\right)}
$$

This equation would give the value of the determinant for $\lambda>1$ in terms of the determinant for $\lambda<1$ provided we know $x_{n}$ for $\lambda<1$.

To proceed further, we switch over to the convention that the root which ensures $c(\pi)>0$ will be chosen and solve the Wiener-Hopf equations

$$
\sum_{m=0}^{n} \bar{G}_{k-m}\left(1 / \lambda_{0}\right) \bar{x}_{m}=\delta_{k, 0} .
$$

The solution for $\bar{x}_{n}$ as found by $\mathrm{Wu}[21]$ is

$$
\bar{x}_{n}=\frac{1}{2 \pi} \int_{0}^{2 \pi} d k e^{i n k} l(k),
$$

with $l(k)$ defined through 22]

$$
\ln l(k)=\sum_{m=1}^{\infty} g_{-m} e^{i m k}-\sum_{m=0}^{\infty} g_{m} e^{-i m k} .
$$

Putting here the expressions of $g_{0}$ and $g_{ \pm m}$ (Eqs. (18), (19)) directly and using (21), we get

$$
\begin{aligned}
\bar{x}_{n}= & \frac{1}{2 \pi} \int_{0}^{2 \pi} d k e^{i n k} \sqrt{1+\lambda^{2}+2 \lambda \cos k} \times \\
& \exp \left[\sum_{m=1}^{\infty} \frac{2}{m} \cos (m \phi) \cos (m k)\right]
\end{aligned}
$$

We rewrite this as

$$
\begin{aligned}
\bar{x}_{n} & =\frac{1}{\pi} \int_{0}^{\pi} d k \cos (n k) \sqrt{1+\lambda^{2}+2 \lambda \cos k} \times \\
& \exp \left[\sum_{m=1}^{\infty} \frac{1}{m}\{\cos (m \phi+m k)+\cos (m \phi-m k)\}\right]
\end{aligned}
$$

and note that the formula (21) may be applied to $\sum(1 / m) \cos (m \phi+m k)$ but not to $\sum(1 / m) \cos (m \phi-m k)$ since the former is convergent in the range of integration while the latter diverges at $k=\phi$. Thus we have,

$$
\bar{x}_{n}=\frac{1}{\pi} \int_{0}^{\pi} d k \frac{\cos (n k) \sqrt{1+\lambda^{2}+2 \lambda \cos k}}{2 \sin \frac{\phi+k}{2}} e^{\mathcal{S}}
$$

where,

$$
\mathcal{S}=\sum_{m=1}^{\infty} \frac{1}{m} \cos (k-\phi) m
$$

For large $n$, the term $\cos (n k)$ oscillates rapidly and the non-trivial contribution to the integral comes only from the points where the rest of the integrand diverges. Hence, it suffices to integrate only over a narrow region (of width $2 \epsilon \ll 1 / n$ ) around the point $k=\phi$ and obtain

$$
\bar{x}_{n}=\frac{1}{2 \pi} \frac{\sqrt{1+\lambda^{2}+2 \lambda \cos (\phi)}}{\sin (\phi)} \int_{-\epsilon}^{\epsilon} \cos (n x+n \phi) e^{\mathcal{S}} d x
$$

where we have taken the smoothly varying part out of the integration and put $x=k-\phi$. Following the FisherHartwig conjecture, we now replace ("heuristically") the upper limits in the sum $\mathcal{S}$ (see Eq. (33)) by $\zeta^{\prime} n$ and note that for $\zeta^{\prime} \sim 1$, we must have $x m \ll 1$ for all $m$ in the range of integration. The sum $\mathcal{S}$ may then be approximated as

$$
\mathcal{S}=\sum_{m=1}^{\zeta^{\prime} n}\left[\frac{1}{m}-\frac{1}{2} x^{2} m\right] .
$$

Substituting Eq. (22) in the first summation, we obtain,

$$
e^{\mathcal{S}}=2 \sqrt{\pi} e^{\gamma_{E}}\left[\frac{\zeta^{\prime} n}{2 \sqrt{\pi}} e^{-x^{2}\left(\zeta^{\prime} n / 2\right)^{2}}\right]
$$


As $n \rightarrow \infty$, the portion within $[\cdots]$ becomes the Dirac delta functon $\delta(x)$ and the expression (34) for $\bar{x}_{n}$ becomes

$$
\bar{x}_{n}=\frac{e^{\gamma_{E}}}{\sqrt{\pi}} \frac{\sqrt{1+\lambda^{2}+2 \lambda \cos (\phi)}}{\sin (\phi)} \cos (n \phi)
$$

The unknown constant $\zeta^{\prime}$ (happily) does not appear in this final expression. Since $\bar{x}_{n}=x_{n}$, Eqs. (12a), (23), and (27) now give our final expression for the correlation function for $\lambda>1$ :

$$
\begin{aligned}
C^{z}(n) & =\operatorname{det}_{n}(\lambda)=(-1)^{n} x_{n}(1 / \lambda) \operatorname{det}_{n+1}(1 / \lambda) \\
& =\frac{A e^{\gamma_{E}}}{\sqrt{\pi} \sin (\phi)}\left(1-\frac{1}{\lambda^{2}}\right)^{1 / 4} \frac{\cos (\pi+\phi) n}{\sqrt{n}} .
\end{aligned}
$$

(The constant $A$ is defined below Eq. (23).) This correlation function decays algebraically with distance and also oscillates with distance with wave-vector $(\pi+\phi)$.

Before we conclude this section, we point out that although Eq.(29) for $\bar{x}_{n}$ as derived by Wu 21] is applicable here, there is a major difference between our case and the one studied by $\mathrm{Wu}$. In our case, $x_{n}$ does not vanish in the limit $n \rightarrow \infty$ and all we want to evaluate is the zero-th order term in $n$. But in Wu's treatment, $\lim _{n \rightarrow \infty} x_{n}$ is zero and the basic job was to evaluate the leading terms for large $n$.

\section{DISCUSSIONS}

(i) If we look at the spin-spin correlation in the ground state of transverse Ising model, then we find that it is only for $\lambda=1$ that the correlation decays algebraically with distance. But for the type of excited states we study (see Eq. (5)), the algebraic behaviour is seen over a larger range, namely, for $\lambda<1$ as well as $\lambda>1$ and any $\phi$ in the range $0<\phi<\pi$.

(ii) For handling the divergent sums that arise in the calculation of correlation function, Fisher-Hartwig prescription is very much useful for the excited states as it was for the ground state. Actually, the index for algebraic decay can be derived from the generating function itself, without any further calculation. Fisher and Hartwig have shown that for generating function having multiple singularities, if $\arg [c(k)]$ jumps by an amount $2 \pi i \alpha_{j}$ at the $j$-th singularity, then the determinant will decay algebraically with distance with an exponent $\sum_{j} \alpha_{j}^{2}$. In our case, the generating function of Eq. (10) has two jumps, at each of which $\alpha_{j}$ is $1 / 2$, and the index is $1 / 2$, obeying this conclusion. Moreover, if we consider an excited state where (in contrast to Eq. (5)) the excited modes extend from $k=\phi_{1}$ to $\phi_{2}$, then (for $\left.\phi_{2} \neq \pi\right)$ there will be four singularities in the generating function, at each of which $\alpha_{j}$ is $1 / 2$. The correlation will then decay as $1 / n$.

\section{Acknowledgments}

The work of one author (AKC) was supported by UGC fellowship.
[1] B.K. Chakrabarti, A. Dutta and P. Sen, Quantum Ising Phases and Transitions in Transverse Ising Models (Springer-Verlag, Berlin, Heidelberg) 1996.

[2] G. Uimin and H. Rieger, Z. Phys. B 101, 597 (1996).

[3] A. Dutta and D. Sen, Phys. Rev. B 67, 094435 (2003).

[4] A. K. Chandra and S. Dasgupta, arXiv:cond-mat/0612144, Phys. Rev. E 75, 021105 (2007).

[5] E. Lieb, T. Schultz and D. C. Mattis, Annals of Phys. 16, 407 (1961).

[6] P. Pfeuty, Annals of Phys. 57, 79 (1970).

[7] D. C. Mattis, The Theory of Magnetism, Vol. II (Springer-Verlag, Berlin, Heidelberg) 1985, Sec. 3.6.

[8] B.M. McCoy, Phys. Rev. 173, 531 (1968).

[9] See Ref [5] Eq. (2.88).

[10] For an excellent elaborate discussion (for a physicist) on Toeplitz determinant and Szego's Theorem, see B.M. McCoy and T.T. Wu, The Two-Dimensional Ising Model (Harvard University Press, Cambridge, Massachusetts) 1973 Chapter X.

[11] M.E. Fisher and R.E. Hartwig, Adv. Chem. Phys. 15,
333 (1968)

[12] A. Bottcher, J. Stat. Phys. 78, 575 (1995).

[13] T. Ehrhardt, Operator Th : Advances and App. 124217 (2001).

[14] McCoy and $\mathrm{Wu}$, 10], pages 186, 244.

[15] McCoy and Wu [10], p. 250; T.T. Wu, Phys. Rev. 149, 380 (1966), see p.381.

[16] McCoy and Wu [10], p. 225.

[17] Fisher and Hartwig [11], p. 338, Theorem 2.

[18] I.S. Gradshteyn and I.M. Ryzhik, Table of Integrals, Series, and Products (Academic Press, New York) 1980 Entry No. (1.448 2).

[19] Fisher and Hartwig's 'conjecture' is however, no longer a conjecture, since it has been proven by E.L. Basor, Indiana Math. J. 28, 975 (1979).

[20] McCoy and Wu, 10], Chapter XI; also in T.T. Wu, Phys. Rev. 149, 380 (1966) .

[21] McCoy and Wu [10], p. 253, Eq. (2.27); Wu 20] Eq. (2.27).

[22] Fisher and Hartwig [11], p. 341, Eq. (42). The $m=0$ term must be added to the second summation there. 